\title{
DIFERENTES PERFIS DE MOTIVAÇÕES E PREOCUPAÇÕES DOS ESTUDANTES DE EDUCAÇÃO FÍSICA
}

\author{
DIFFERENT PROFILES IN TERMS OF MOTIVATION AND CONCERNS OF \\ PHYSICAL EDUCATION STUDENTS
}

\section{DIFERENTES PERFILES DE MOTIVACIONES Y PREOCUPACIONES DE LOS ESTUDIANTES DE EDUCACIÓN FÍSICA}

\author{
Tiago Garcia Batilani", Isabella Caroline Belem ${ }^{* *}$, Jorge Both ${ }^{* *+}$
}

Palavras chave:

Motivação.

Educação superior.

Renda.

Educação Física.
Resumo: 0 objetivo da investigação foi identificar os diferentes perfis de alunos do curso de Licenciatura em Educação Física considerando a motivação e preocupação dos discentes. No estudo quantitativo e transversal participaram 53 estudantes vinculados ao Estágio Curricular Supervisionado. Os instrumentos de pesquisa foram: Escala de Motivação Inicial e Apoio Pedagógico, Escala de Preocupações Pedagógica e Questionário Sociodemográfico. Os resultados evidenciaram três grupos de acadêmicos. O grupo I era composto por estudantes desmotivados, preocupados apenas com a tarefa, com pouco tempo para os estudos e renda insuficiente. Os grupos II e III apresentaram maior motivação. Entretanto, o grupo II possuía maior índices de preocupação e renda insuficiente e menor tempo dedicado para os estudos, enquanto que o grupo III possuía menores índices de preocupação e renda insuficiente e maior tempo dedicado aos estudos. Assim, conclui-se que os aspectos sociodemográficos são fatores intervenientes nos perfis de motivação e preocupação de estudantes.

Keywords:

Motivation.

Education higher.

Income.

Physical Education.

Palabras clave: Motivación

Educación superior. Renta.

Educación Física.
Abstract: The aim of this study was to identify different profiles of Physical Education teacher students considering their motivation and concerns. A total of 53 students in Supervised Internship participated in this quantitative and cross-sectional study. The research instruments included: Initial Motivation and Pedagogical Support Scale; Teacher's Concerns Questionnaire; and Sociodemographic Questionnaire. The results showed three groups of students. Group I included unmotivated students concerned only with the task at hand and with little time for studies and insufficient income. Students in Groups II and III showed higher motivation. However, members of Group II showed higher levels of concern, insufficient income and less time dedicated to studies, while Group III members had lower levels of concern, insufficient income and more time dedicated to studies. Therefore, the study finds that sociodemographic aspects are intervening factors for students' motivation and concern profiles.

Resumen: El objetivo de la investigación fue identificar los diferentes perfiles de alumnos de un curso de Licenciatura en Educación Física según su motivación y preocupación. Fue realizado un estudio cuantitativo y transversal con 53 estudiantes matriculados en la materia Práctica Docente Supervisada. Los instrumentos de investigación fueron: Escala de Motivación Inicial y Apoyo Pedagógico, Escala de Preocupaciones Pedagógicas y Cuestionario Sociodemográfico. Los resultados mostraron tres grupos académicos. El grupo I estaba formado por estudiantes desmotivados, preocupados solo con la tarea, con poco tiempo para estudiar y renta insuficiente. Los grupos II y III presentaron motivación mayor. Sin embargo, el grupo II tenía índices de preocupación mayores, renta insuficiente y tiempo de dedicación menor para los estudios. El grupo III poseía índices de preocupación menores, renta insuficiente y más tiempo para estudiar. Se concluye que los aspectos sociodemográficos son factores que inciden en los perfiles de motivación y preocupación de estudiantes.
*Faculdade Intermunicipal do Noroeste do Paraná. Loanda, PR, Brasil. Universidade Estadual de Londrina. Londrina, PR, Brasil.

E-mail: tiagogarciabatilani@ hotmail.com

**Universidade Paranaense. Umuarama, PR, Brasil; Centro Universitário de Maringá. Maringá, PR, Brasil. Universidade Estadual de Londrina. Londrina, PR, Brasil. E-mail: isabellacbelem@gmail.com

*** Universidade Estadual de Londrina. Londrina, PR, Brasil.

E-mail: jorgeboth@yahoo.com.br

Recebido em: 12-07-2017 Aprovado em: 26-05-2018

$\mathrm{DOl}:$ http://dx.doi.org/10.22456/1982-8918.74947 (c) (1) () Licence 


\section{INTRODUÇÃOO}

Atualmente no Brasil existem 2.153 cursos de graduação em Educação Física (EF), sendo $1.578 \mathrm{em}$ licenciatura, com mais de 149 mil matrículas, na qual a maioria é do gênero masculino e vinculada a instituições privadas. Ao considerar o estado do Paraná, observa-se a existência de 39 cursos de graduação em licenciatura em EF, o que faz desta unidade federativa a quarta colocada na oferta de cursos desta habilitação no âmbito nacional (MARTINS, 2015; BRASIL, 2014).

O processo de formação do docente é constituído por fases, iniciando-se na graduação, seguida pela entrada no mercado de trabalho. A formação docente é um processo contínuo, sendo que a formação inicial é base do processo, uma vez que o acadêmico irá começar a adquirir conhecimentos científicos e pedagógicos para desenvolver as competências necessárias para atuar na carreira docente. Deste modo o Estágio Curricular Supervisionado (ECS) é um fator essencial para o processo de socialização profissional (VIEIRA et al., 2006; SILVA PIOVANI et al., 2012).

O momento do ECS é importante na formação profissional e proporciona ao aluno condições de vivenciar situações de aprendizagem relacionadas à realidade de trabalho no ambiente escolar (VIEIRA et al. 2006; FOLLE et al., 2009; IZA; SOUZA NETO, 2015). Para muitos discentes o primeiro contato com a realidade pode proporcionar um "choque", pois 0 discente se depara com problemas como: indisciplina, falta de interesse dos alunos, escola com espaço físico inadequado, falta de materiais para as atividades pedagógicas, insegurança para ministrar os conteúdos e dificuldades na elaboração do planejamento das atividades (RIBEIRO et al., 2015).

O contato com a realidade escolar proporcionado pela disciplina de ECS pode motivar os acadêmicos sobre a futura profissão. Discentes mais motivados tendem a ter melhor aprendizagem e desempenho nas atividades acadêmicas, tornando-se mais participativos e dedicados para a retenção e domínio do conteúdo apresentado (GUIMARÃES; BORUCHOVITCH, 2004).

Para Lens, Matos e Vansteenkiste (2008), motivação é fator fundamental para discentes durante o processo de ensino e aprendizagem da disciplina de ECS. A motivação pode vir de fatores intrínsecos (pessoais) ou extrínsecos (ambientais). A motivação intrínseca para a escolha do curso de licenciatura em EF na maioria das vezes pode estar relacionada à prática de atividades físicas, sejam elas ligadas ao ambiente escolar ou não, como atividades de lazer ou de esporte rendimento. Outro fator importante ligado à motivação acadêmica está relacionado à permanência e conclusão do curso. Os discentes se identificam com os conteúdos curriculares presentes ao longo do curso e ao iniciarem a prática dos estágios passam a lecionar esses conteúdos com êxito, sentindo-se mais motivados para ingressar no mercado de trabalho (FIGUEIREDO, 2008).

A motivação extrínseca está relacionada com fatores ambientais presentes na vida pessoal e acadêmica do estudante. Durante sua vida acadêmica vários fatores podem influenciar sua motivação, dentre eles os professores que ministram a disciplina de ECS, os quais serão os responsáveis pelo primeiro contato com a prática em ambiente escolar (JOLY; PRATES, 2011). 
O professor da disciplina ECS pode motivar os acadêmicos em dois aspectos. Primeiro, quando os alunos percebem que o docente se esforça para adaptar ou criar estratégias de ensino que melhorem o aprendizado, diminuindo chances de possíveis fracassos e decepções. Segundo, quando presta auxílio aos acadêmicos, ao acompanhá-los e auxiliá-los durante a prática na resolução dos problemas encontrados (MARCON; NASCIMENTO; GRAÇA, 2007; LENS; MATOS; VANSTEENKISTE, 2008).

Em alguns casos os primeiros contatos com a realidade podem levar ao encontro de conflitos e problemas, podendo causar desmotivação nos acadêmicos. Os problemas vivenciados durante o ECS, quando não abordados corretamente, podem se tornar preocupações que geram sensações de incerteza e insegurança, pois os estudantes-estagiários ainda não estão preparados para assumir as responsabilidades de professores (FARIAS et al., 2008).

Segundo a Teoria de Fuller sobre preocupações de professores (FULLER, 1969; FULLER; BROWN, 1975), algumas inquietações surgem no período da graduação, quando os acadêmicos têm os primeiros contatos com a realidade escolar através da disciplina de ECS. Estas preocupações são nomeadas em consigo, tarefa e impacto da tarefa. A preocupação consigo está relacionada com a sobrevivência do acadêmico dentro da sua atuação docente, relacionada ao momento que ministrará aula na comunidade escolar. Preocupação com a tarefa está associada à execução do trabalho relacionado ao planejamento e execução das atividades propostas. Preocupação com o impacto da tarefa está vinculada ao resultado final das atividades de ensino, ou seja, o quanto as tarefas desenvolvidas durante as aulas podem afetar o aluno (OLSEN; HEYSE, 1990; FARIAS et al., 2008; SILVA PIOVANI et al., 2012).

Estudos sobre ECS em EF têm sido realizados a fim de investigar as preocupações pedagógicas (FARIAS et al. 2008), a atuação do professor colaborador (IZA; SOUZA NETO, 2015; LENS; MATOS; VANSTEENKISTE, 2008) e a influência da atividade de estágio na formação do futuro professor (MARCON; NASCIMENTO; GRAÇA, 2007). Nota-se uma lacuna na literatura quando se trata de investigações que buscam analisar os diferentes tipos de grupos de estudantes-estagiários que existem no momento da realização do ECS considerando aspectos motivacionais e preocupações dos discentes. Nesse sentido, o objetivo do estudo foi identificar os diferentes perfis de alunos considerando os aspectos associados à motivação $e$ a preocupação dos discentes.

\section{MATERIAIS E MÉTODOS}

A pesquisa descritivo-exploratória, de abordagem quantitativa e de corte transversal teve como amostra 53 acadêmicos do curso de licenciatura em EF de uma instituição privada de ensino superior localizada na microrregião do extremo noroeste do Paraná, a qual está a $585 \mathrm{~km}$ de distância da cidade de Curitiba, capital do estado. Destaca-se que os discentes cursavam a disciplina de ECS e tinham média de idade de 25,0 anos (Desvio Padrão de 4,80 anos).

O questionário sociodemográfico construído para pesquisa buscou identificar as características: Sexo, Idade, Ano do Curso, Estado Civil, Possui Dependentes, Renda, Experiência no Estágio Remunerado, Vínculo Empregatício, Horas de Trabalho, Experiência como Atleta e Tempo Dedicado ao Estudo. 
Para avaliar a motivação dos acadêmicos foi utilizada a Escala de Motivação Inicial e Apoio Pedagógico (JESUS, 1996). Esse questionário é dividido em quatro dimensões: Motivação Inicial, que compreende sete questões e suas respostas variam em uma escala likert de cinco pontos (1 "nenhuma" a 5 "muita"); Modelo de Formação Educacional, composto por dez itens que variam em uma escala likert de sete pontos (1 "discordo totalmente" a 7 "concordo totalmente"); Resultados Profissionais, formada por dez itens dispostos em escala likert de sete pontos (1 "discordo totalmente" a 7 "concordo totalmente"); Apoio no Estágio, que contém sete questões que variam em uma escala likert de cinco pontos (1 "malsucedido" a 5 "bem-sucedido").

A Escala de Preocupações Pedagógicas dos professores traduzida e validada para a realidade brasileira no estudo de Costa (2013) é a adaptação do instrumento desenvolvido por McBride (1984), Teachers Concerns Questionnaire, o qual foi empregado para avaliar preocupações de professores. A escala é composta por 15 questões divididas nas dimensões: Consigo, Tarefa e Impacto da Tarefa. Para cada item existe uma escala likert de cinco pontos que podem variar de "Não preocupado" a "Extremamente preocupado".

Após a autorização da instituição de ensino superior, foram agendadas as coletas com os professores e os acadêmicos, bem como foram explanados os objetivos da pesquisa. As coletas ocorreram ao final das aulas e todos os sujeitos assinaram o termo de consentimento livre e esclarecido. A aplicação dos instrumentos foi realizada pelo pesquisador responsável de forma individual e teve duração média de 30 minutos.

Para a descrição dos resultados utilizou-se mediana (Md), intervalo interquartil (Q1-Q3), frequência absoluta (n) e relativa (\%). Para analisar os aspectos motivacionais eas preocupações dos estudantes empregaram-se os testes de Friedman e de comparação múltipla de Dunn. A análise multivariada dos dados foi realizada por meio da Análise de Cluster. Destaca-se que foi realizado o teste de Anova one-way para identificar, por meio do critério do $\mathrm{R}^{2}$ ( $\mathrm{R}$ quadrado), o número adequado de grupos que deveriam ser considerados na análise (MAROCO, 2007). Ressalta-se que os resultados das médias das dimensões das escalas utilizadas no estudo foram transformados para uma escala de 0 a 100 pontos para normatizar as respostas dos instrumentos. Após a identificação dos grupos foram utilizados os testes de Kruskal-Wallis e Qui-Quadrado para analisar a relação existente entre os grupos identificados na Análise de Cluster com a motivação, as preocupações e as variáveis sociodemográficas. Ressalta-se que foi empregado o teste de comparação múltipla de Dunn para comparar pormenorizadamente as análises de Kruskal-Wallis que obtiveram associação significativa. Por fim, destaca-se que o nível de significância adotado no estudo foi de $95,0 \%(p \leq 0,05)$.

\section{RESULTADOS}

Os resultados evidenciaram que a maioria do grupo era do sexo masculino ( $n=28$; $52,8 \%)$, cursava a $3^{\text {a }}$ série ( $\left.n=28 ; 52,8 \%\right)$, tinha companheiros ( $\left.n=27 ; 50,9 \%\right)$ e não tinha filhos $(n=45 ; 84,9 \%)$. Quanto aos aspectos financeiros e empregatícios observou-se que a maior parte dos discentes não tinha experiência com estágio remunerado $(n=38 ; 71,7 \%)$, possuía vínculo empregatício ( $n=37 ; 69,8 \%$ ) e não eram $(n=47 ; 88,3 \%)$ e nunca foram atletas $(n=32$; $60.4 \%$ ) de equipes de esporte de rendimento (Tabela 1). 
Tabela 1 - Perfil sociodemográfico dos acadêmicos de licenciatura em Educação Física.

\begin{tabular}{lcc}
\hline Variável & Categoria & $\mathrm{n}(\%)$ \\
\hline \multirow{2}{*}{ Sexo } & Masculino & $28(52,8)$ \\
& Feminino & $25(47,2)$ \\
\hline \multirow{2}{*}{ Ano do Curso } & $3^{\circ}$ Ano & $28(52,8)$ \\
& $4^{\circ}$ Ano & $25(47,2)$ \\
\hline \multirow{2}{*}{ Estado Civil } & Com companheiro & $27(50,9)$ \\
\hline \multirow{2}{*}{ Possui Dependentes } & Sem companheiro & $26(49,1)$ \\
\hline \multirow{2}{*}{ Renda Suficiente } & Sem filhos & $45(84,9)$ \\
& Com filhos & $8(15,1)$ \\
\hline Experiência no Estágio & Suficiente & $28(52,8)$ \\
Remunerado & Não suficiente & $25(47,2)$ \\
\hline \multirow{2}{*}{ Vínculo empregatício } & Sim & $15(28,3)$ \\
& Não & $38(71,7)$ \\
\hline \multirow{2}{*}{ É atleta } & Sim & $37(69,8)$ \\
\hline \multirow{2}{*}{ Foi atleta } & Não & $16(30,2)$ \\
\hline
\end{tabular}

Fonte: Produzido pelos autores

Ao avaliar os resultados dos constructos da motivação e das preocupações dos acadêmicos em licenciatura em EF (Tabela 2), observou-se associação significativa no constructo da Motivação e Apoio Pedagógico ( $\mathrm{p}<0,001$ ), em que a dimensão Apoio no Estágio $(\mathrm{Md}=89,3)$ demonstrou ser 0 aspecto mais positivo quando comparado às dimensões Motivação Inicial e Apoio Pedagógico (Md=67,9) ( $\mathrm{p}<0,001)$, Modelo de Formação Educacional $(\mathrm{Md}=70,0)$ $(\mathrm{p}<0,001)$ e Resultados Profissionais $(\mathrm{Md}=70,0)(\mathrm{p}<0,001)$. Ressalta-se que 0 constructo das Preocupações $(p<0,001)$ também evidenciou associação significativa, sendo que as dimensões Consigo $(M d=60,0)(p=0,020)$ e Impacto da Tarefa $(M d=60,0)(p<0,001)$ apresentaram escores mais elevados estatisticamente que a dimensão Tarefa $(\mathrm{Md}=50,0)$.

Tabela 2 - Comparação das dimensões dos constructos de Motivação e Apoio Pedagógico e Preocupações dos acadêmicos de licenciatura em Educação Física.

\begin{tabular}{llc}
\hline Constructos & Mediana (Q1-Q3) & $\mathbf{p}^{*}$ \\
\hline Motivação e Apoio Pedagógico & & $<0,001$ \\
\hline Motivação Inicial e Apoio Pedagógico & $67,9(57,1-78,6)^{\mathrm{a}}$ & \\
Modelo de Formação Educacional & $70,0(50,0-78,3)^{\mathrm{a}}$ & \\
Resultados Profissionais & $70,0(51,7-83,3)^{\mathrm{a}}$ & \\
Apoio no Estágio & $89,3(78,6-100,0)^{\mathrm{b}}$ & $<0,001$ \\
\hline Preocupações & & \\
\hline Consigo & $60,0(45,0-80,0)^{\mathrm{a}}$ & \\
Tarefa & $50,0(35,0-60,0)^{\mathrm{b}}$ & \\
Impacto da Tarefa & $60,0(45,0-85,0)^{\mathrm{a}}$ & \\
\hline
\end{tabular}


Para determinar o número de grupos empregou-se após a Análise de Cluster o método do R-quadrado (Gráfico 1). A partir dos cálculos foi possível identificar que o número de grupos aceitável para o estudo estava entre dois e três, isto porque a variabilidade foi maior entre estes Clusters. Entretanto, a flexão da linha do gráfico iniciou-se com maior propriedade com três Clusters.

Figura 1 - Critério de R-Quadrado para retenção de Clusters.

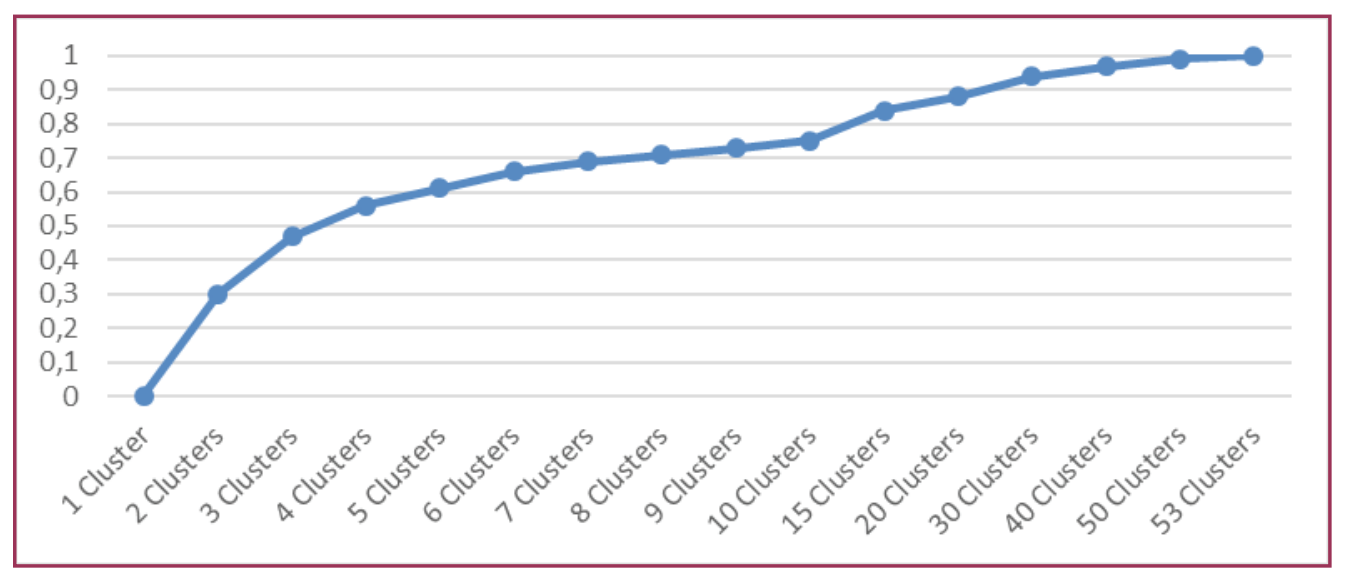

Fonte: Produzido pelos autores

A distribuição do número de alunos por Cluster ficou estabelecida da seguinte maneira: 25 alunos no Cluster II (47,2\%), 14 alunos no Cluster I (26,4\%) e 14 alunos no Cluster III $(26,4 \%)$ (Tabela 4). Ao comparar as motivações e preocupações de acordo com os três grupos estabelecidos na análise de Clusters observaram-se diferenças significativas em todas as dimensões dos constructos $(p \leq 0,033)$.

Tabela 4 - Associação entre motivação e apoio pedagógico e preocupação conforme os grupos identificados na análise de Clusters.

\begin{tabular}{lcccc}
\hline \multirow{2}{*}{ Constructos e Dimensões } & \multicolumn{3}{c}{ Cluster } & \multirow{2}{*}{$\mathbf{p}^{*}$} \\
\cline { 2 - 4 } & $\mathbf{I}(\mathbf{n}=\mathbf{1 4})$ & $\mathbf{I I}(\mathbf{n}=\mathbf{2 5})$ & $\mathbf{I I I}(\mathbf{n}=\mathbf{1 4})$ & \\
\cline { 2 - 4 } & $\mathbf{M d}(\mathbf{Q 1}-\mathbf{Q} \mathbf{3})$ & $\mathbf{M d}(\mathbf{Q 1}-\mathbf{Q} \mathbf{3})$ & $\mathbf{M d}(\mathbf{Q 1}-\mathbf{Q} 3)$ & \\
\hline Motivação e Apoio Pedagógico & & & & \\
\hline Motivação Inicial & $53,6(39,3-71,4)$ & $71,4(64,3-82,1)$ & $73,2(64,3-78,6)$ & $\mathbf{0 , 0 3 3}$ \\
Formação Educacional & $39,2(36,7-65,0)$ & $76,7(55,0-83,3)$ & $75,9(69,3-81,7)$ & $<0,001$ \\
Resultados profissionais & $48,4(36,7-65,0)$ & $68,3(66,7-80,0)$ & $84,2(83,3-91,7)$ & $<0,001$ \\
Apoio Estágio & $75,0(71,4-82,1)$ & $89,3(85,7-100,0)$ & $92,9(82,1-100,0)$ & $\mathbf{0 , 0 0 2}$ \\
\hline Preocupações pedagógicas & & & & \\
\hline Consigo & $50,0(50,0-55,0)$ & $80,0(70,0-90,0)$ & $32,5(15,0-40,0)$ & $<0,001$ \\
Tarefa & $50,0(40,0-60,0)$ & $60,0(50,0-75,0)$ & $27,5(20,0-40,0)$ & $<0,001$ \\
Impacto & $50,0(35,0-55,0)$ & $85,0(75,0-95,0)$ & $45,0(30,0-50,0)$ & $<0,001$ \\
\hline
\end{tabular}

*Probabilidade estimada teste de Kruskal-Wallis

Fonte: Produzido pelos autores

$\mathrm{Na}$ avaliação do constructo da Motivação e Apoio Pedagógico, observou-se que os acadêmicos dos Clusters II e III apresentam índices mais positivos nas dimensões: Motivação Inicial (Cluster II - $p=0,018$; Cluster III - $p=0,024)$, Formação Educacional (Cluster II $-p<0,001$; Cluster III - $p<0,001$ ) e Apoio no Estágio (Cluster II - $p=0,002$; Cluster III - $p=0,002$ ) quando comparados com os discentes do Cluster I. Ressalta-se que na dimensão Resultados Profissionais evidenciaram-se diferenças significativas nos três grupos, sendo que o Cluster 
III apresentou o maior índice quando comparados os Clusters I $(p<0,001)$ e II $(p=0,015)$. Além disso, o Cluster I demonstrou menor escore na dimensão Resultados Profissionais quando comparado com o Cluster II ( $p=0,005)$. Por outro lado, na comparação entre os Clusters conforme as Preocupações, constatou-se que os discentes dos Clusters I e III eram menos preocupados com as dimensões Consigo (Cluster I $-p<0,001$; Cluster III $-p=0,022$ ) e Impacto da Tarefa (Cluster I $-p<0,001$; Cluster III $-p<0,001$ ) que os alunos do Cluster II. Por outro lado, os discentes dos Clusters I $(p=0,011)$ e II $(p<0,001)$ apresentaram maiores índices de preocupação com a dimensão Tarefa quando comparados com os discentes do Cluster III.

Ao avaliar os Clusters conforme as variáveis sociodemográficas (Tabelas 5 e 6), foram identificadas diferenças significativas entre os grupos de alunos e as variáveis Tempo de Estudo $(p=0,018)$ e Renda Suficiente $(p=0,014)$.

Tabela 5 - Diferença entre dados sociodemográficos dos acadêmicos de licenciatura em Educação Física segundo os grupos identificados na análise de Cluster.

\begin{tabular}{|c|c|c|c|c|}
\hline \multirow{3}{*}{$\begin{array}{c}\text { Variáveis } \\
\text { Sociodemográficos }\end{array}$} & \multicolumn{3}{|c|}{ Cluster } & \multirow{3}{*}{$p^{*}$} \\
\hline & $I(n=14)$ & II $(n=25)$ & III $(n=14)$ & \\
\hline & $\operatorname{Md}(\mathbf{Q} 1-Q 3)$ & $M d(Q 1-Q 3)$ & $M d(Q 1-Q 3)$ & \\
\hline Idade & $25,0(23,5-29,5)$ & $23,0(21,0-29,5)$ & $23,0(22,0-26,0)$ & 0,298 \\
\hline Horas de trabalho & $25,0(9,0-40,0)$ & $39,0(6,0-41,0)$ & $30,0(12,0-40,0)$ & 0,745 \\
\hline Tempo de estudo & $3,0(1,5-4,0) a$ & $3,0(2,0-5,0) a$ & $5,0(4,0-8,0) b$ & 0,018 \\
\hline
\end{tabular}

*Probabilidade estimada teste de Kruskal-Wallis Fonte: Produzido pelos autores

Tabela 6 - Associação entre as variáveis sociodemográficos dos acadêmicos de licenciatura em Educação Física segundo os grupos identificados na análise de Cluster.

\begin{tabular}{|c|c|c|c|c|}
\hline \multirow{3}{*}{$\begin{array}{l}\text { Variáveis } \\
\text { Sociodemográficos }\end{array}$} & \multicolumn{3}{|c|}{ Clusters } & \multirow{3}{*}{$p^{*}$} \\
\hline & $I(n=14)$ & II $(n=25)$ & III $(n=14)$ & \\
\hline & $\mathrm{N}(\%)$ & $\mathrm{N}(\%)$ & $N(\%)$ & \\
\hline \multicolumn{5}{|l|}{ Sexo } \\
\hline Masculino & $08(57,1)$ & $10(40,0)$ & $10(71,4)$ & 0,157 \\
\hline Feminino & $06(42,9)$ & $15(60,0)$ & $04(28,6)$ & \\
\hline \multicolumn{5}{|l|}{ Renda suficiente } \\
\hline Sim & $05(35,7)$ & $11(44,0)$ & $12(85,7)$ & 0,014 \\
\hline Não & $09(64,3)$ & $14(56,0)$ & $02(14,3)$ & \\
\hline \multicolumn{5}{|l|}{ ESO Remunerado } \\
\hline Sim & $03(21,4)$ & $07(28,0)$ & $05(35,7)$ & 0,703 \\
\hline Não & $11(78,6)$ & $18(72,0)$ & $09(64,3)$ & \\
\hline \multicolumn{5}{|l|}{ Vínculo empregatício } \\
\hline Sim & $09(64,3)$ & $18(72,0)$ & $10(71,4)$ & 0,871 \\
\hline Não & $05(35,7)$ & $07(28,0)$ & $04(28,6)$ & \\
\hline \multicolumn{5}{|l|}{ Filhos } \\
\hline Sim & $03(21,4)$ & $04(16,0)$ & $01(7,1)$ & 0,564 \\
\hline Não & $11(78,7)$ & $21(84,0)$ & $13(92,9)$ & \\
\hline \multicolumn{5}{|l|}{ Atleta } \\
\hline Sim & $0(0,0)$ & $04(16,0)$ & $02(14,3)$ & 0,293 \\
\hline Não & $14(100,0)$ & $21(84,0)$ & $12(85,7)$ & \\
\hline \multicolumn{5}{|l|}{ Foi atleta } \\
\hline Sim & $06(42,9)$ & $08(32,0)$ & $07(50,0)$ & 0,522 \\
\hline Não & $08(57,1)$ & $17(68,0)$ & $07(50,0)$ & \\
\hline
\end{tabular}


$\mathrm{Na}$ avaliação sobre o tempo de estudo dos alunos (Tabela 5) constatou-se que os discentes do Cluster III (5,0 horas) demonstraram maior tempo dedicado ao estudo que os Clusters I (3,0 horas) e II (3,0 horas). Sobre a Renda (Tabela 6) observou-se que a maioria dos alunos do Cluster III (85,7\%) apresentou renda suficiente para suas necessidades básicas. Por outro lado, a maioria dos discentes dos Clusters I (64,3\%) e II (56,0\%) informou que não possuía renda suficiente para suas necessidades básicas.

\section{DISCUSSÃO}

Ao avaliar os resultados das variáveis sociodemográficas dos discentes, observouse que os aspectos que mais chamaram a atenção foram: pouca experiência no estágio remunerado, grande vínculo com atividades laborais fora da área de EF, pouca experiência como atletas e não tinham filhos. Evidências semelhantes foram encontradas no Relatório do Exame Nacional de Desempenho dos Estudantes dos cursos de Licenciatura em EF, em que grande parte dos discentes tinha características problemáticas relacionadas à renda familiar e de sobrevivência no local em que o curso era ofertado, como: renda de 1,5 a 3 salários mínimos por mês, responsáveis ou corresponsáveis com o sustento da família e recebimento de ajuda financeira da família ou de outras pessoas para pagar os estudos (BRASIL, 2014).

Assim, a preferência pelo estágio supervisionado remunerado fora da área de EF pode estar associada à possibilidade de adquirir renda mensal maior com a qual é possível cobrir despesas. A pouca experiência em estágio remunerado na área de EF pode estar associada ao tempo disponível dos discentes. Observa-se nos resultados que muitos acadêmicos possuíam empregos e estudavam durante o período noturno (ver Tabela 1), o que pode resultar no pouco tempo para realizarem estágios remunerados na área de EF.

0 baixo número de discentes que tinham filhos pode estar relacionado com as transformações sociais, culturais e econômicas, o que faz com que o planejamento familiar gere menor número de filhos devido à elevada carga de compromissos com que o casal arca para o sustento dos dependentes (SCAVONE, 2001).

Sobre a experiência no ambiente esportivo, estudos (GOMES; QUEIRÓS; BATISTA, 2014; ISRAEL; DUARTE, 2000) observaram que a escolha do curso de EF pelos alunos ocorreu pelo vínculo com o esporte de rendimento. Entretanto, para o grupo avaliado a escolha do curso de EF não teve relação com o fato dos acadêmicos terem alguma vivência como atletas. A opção pelo curso de graduação em EF para este grupo de alunos pode estar relacionada às boas lembranças proporcionadas pela prática de atividades físicas vinculadas ao caráter lúdico (FIGUEIREDO, 2008).

Em relação à motivação inicial para a profissão docente, constatou-se que as dimensões: Motivação Inicial, Modelos de Formação e Resultados Profissionais foram importantes para os acadêmicos. Porém o Apoio no Estágio demonstrou ser a dimensão com maior importância. O Apoio no Estágio está relacionado aos primeiros contatos com a realidade da docência no ambiente escolar. Este contato com a realidade da docência se torna fator muito importante aos acadêmicos e demonstra a verdadeira realidade do trabalho do docente no ambiente escolar, o que pode servir como preparação para os discentes, colocando à prova o conhecimento adquirido na graduação. 
Muitos acadêmicos, ao ingressarem no curso de licenciatura em EF, trazem consigo a impressão equivocada de que lecionar é fácil. Ao se depararem com as vivências da realidade do professor esta concepção se altera (GRAÇA, 2014). O contato com a realidade proporcionado pela disciplina de ECS apresenta possíveis dificuldades que os estudantes podem ter ao ingressar na profissão, que vão desde a falta de estrutura e materiais da escola a questões de comportamento dos alunos (QUEIRÓS, 2014). Neste momento do aprendizado o auxílio do professor supervisor do curso superior e do docente colaborador da escola é imprescindível. Quando acompanhados durante este período, os discentes sentem-se amparados, o que pode diminuir angústias e vulnerabilidades, bem como pode induzir o aluno a uma experiência positiva da profissão (SOUZA NETO; SARTI; BENITES, 2016).

Em relação às preocupações dos acadêmicos, constatou-se que eles estavam preocupados com questões vinculadas às dimensões Consigo e Impacto da Tarefa. Estudos com estudantes-estagiários de EF brasileiros (FARIAS et al., 2008), portugueses (MATOS et al., 1991) e uruguaios (SILVA PIOVANI et al., 2012) obtiveram resultados semelhantes.

A Teoria das Preocupações de Fuller (1969) aponta que há uma evolução das preocupações, sendo que o professor iniciante na carreira tem ênfase na preocupação Consigo. Profissionais com um pouco mais de experiência possuem maior preocupação com a realização das Tarefas. Docentes com competências profissionais estabelecidas possuem ênfase na preocupação com o Impacto da Tarefa. Entretanto, o comportamento dos resultados neste estudo e em outros de diferentes ambientes culturais não foi o mesmo (MATOS et al., 1991; FARIAS et al., 2008; SILVA PIOVANI et al., 2012). Observou-se que os acadêmicos apresentaram maior preocupação Consigo, o que parece ser comum durante esta fase. Fuller (1969) aponta que professores em início de carreira tendem a apresentar preocupações Consigo de forma mais acentuada do que as demais, o que está relacionado com as experiências de sobrevivência desta etapa da trajetória docente, sendo que as preocupações se alteram de acordo com a etapa em que os docentes se encontram. Nesse sentido, Ribeiro et al. (2015) apontam que estudantes na formação inicial de EF também podem apresentar estas preocupações encontradas em docentes em início de carreira.

De fato, o acadêmico encontra-se em um período de transição marcado por dois fatores. $O$ primeiro fator está relacionado à preocupação em concluir o curso. $O$ segundo fator são as incertezas em relação ao ingresso no mercado de trabalho. Ao se deparar com a realidade escolar durante a disciplina de ECS, não se sente apto a enfrentar a docência, nem a planejar as atividades. Essa preocupação com o planejamento deve-se ao impacto que as atividades desenvolvidas podem causar sobre os alunos, forçando os discentes a se tornarem reflexivos sobre as condições sociais, econômicas e culturais dos alunos em seus planejamentos (SILVA PIOVANI et al. 2012).

A preocupação mais elevada com o Impacto da Tarefa pode ser consequência da maior inquietação quanto às condições sociais dos alunos com as quais os estagiários se deparam durante a realização dos seus estágios, e que acabam por interferir em seu cotidiano. Isto porque, em sua maioria, os acadêmicos tendem a realizar seus estágios em escolas públicas, que muitas vezes se encontram em periferias (FARIAS et al., 2008).

No entanto, observou-se baixa preocupação com a Tarefa por parte dos entrevistados. O estudo de Ribeiro et al. (2015) verificou que acadêmicos que realizaram os estágios pela primeira vez apresentaram menor preocupação com a Tarefa, o que pode ocorrer devido ao 
momento do curso de graduação em que o estagiário se encontra, visto que as preocupações podem se alterar: enquanto algumas preocupações diminuem, outras surgem devido às experiências adquiridas ao longo da realização do estágio. Silva Piovani e Both (2014) também constataram baixa preocupação com a dimensão Tarefa, o que parece ser influenciado pelo domínio e segurança dos estudantes ao executarem suas atividades durante os estágios, as quais podem sofrer influência da falta de domínio da gestão da turma, falta de materiais, número de alunos, entre outros fatores. Como aponta Fuller (1969), preocupações com as tarefas são atingidas quando aumentam os anseios acerca do planejamento das atividades, execução do conteúdo a ser ministrado, número de alunos na turma e quantidade de material.

Ao avaliar conjuntamente os aspectos motivacionais e de preocupação dos discentes de EF foi possível identificar três grupos homogêneos, sendo que o primeiro evidenciou preocupação com a tarefa e baixa motivação. O segundo grupo apresentou elevados índices de motivação e preocupação. 0 terceiro grupo demonstrou baixa preocupação e elevada motivação. Associado a estas características o Cluster III evidenciou maior tempo dedicado ao estudo e renda suficiente para suas necessidades básicas, enquanto os Clusters I e II evidenciaram características mais frágeis frente ao tempo dedicado ao estudo e renda.

Nesse sentido, aspectos relacionados à renda e tempo dedicado ao estudo podem interferir na motivação dos alunos, pois o terceiro grupo possuía em sua maioria alunos que dedicavam menor tempo ao trabalho, permitindo maior tempo aos estudos. Por outro lado, os discentes apresentavam baixos índices de preocupação nas suas atividades no estágio, o que pode ser associado ao maior tempo dedicado ao estudo, o que pode ofertar maior sentimento de segurança dos alunos deste grupo nas suas ações do estágio. Observou-se que os estudantes do Cluster III se distanciam das características sociodemográficas de renda da maioria dos entrevistados. Os resultados deste grupo são contraditórios à maioria dos alunos que realizam curso de licenciatura em EF da IES investigada e do Relatório do Exame Nacional de Desempenho dos Estudantes, em que a maioria dos discentes apresenta baixa renda, o que pode impactar no tempo dedicado ao estudo fora do ambiente universitário (BRASIL, 2014).

Assim, constata-se que o problema com a renda pode ter influenciado os resultados dos Clusters I e || frente à motivação e preocupação. Discentes do segundo grupo têm o comportamento mais motivado e preocupado com as atividades do estágio, pois podem conceber que sua formação acadêmica pode ser uma possibilidade de melhorar suas condições de vida futuramente. A maioria dos empregos da região onde os acadêmicos residem não proporciona estabilidade empregatícia e financeira, pois são atividades formais no comércio ou na área rural (BRASIL, 2016). Aliado a este fato, o momento em que os discentes estavam no curso pode ser um fator que pode gerar expectativa, pois nos anos finais da graduação existe 0 aumento das atividades acadêmicas vinculadas aos estágios e trabalho de conclusão de curso (GUEDES; SANTOS; LOPES, 2006).

O Cluster I demonstrou as características dos discentes que não se identificaram com o curso. Ressalta-se que estudantes que não se identificam com o curso normalmente apresentam dúvidas se realmente ingressarão no mercado de trabalho da futura profissão (BARDAGl; HUTZ, 2010). Associado a este fator, destaca-se que a microrregião do extremo noroeste do Paraná possui apenas a faculdade investigada que oferta cursos de graduação, sendo que a IES iniciou suas atividades no final do ano de 1999 (PARANÁ, 1999) e atualmente oferta licenciatura em EF e mais três cursos de graduação de forma presencial. Assim, a falta 
de opções de cursos de graduação e de condições financeiras para migrar para outros centros universitários faz com que o discente efetive a matrícula em um curso de graduação com 0 qual não tem afinidade. Tal situação pode gerar pouca motivação para o estudo, fazendo que o discente se preocupe em realizar as tarefas básicas, diminuindo o tempo dedicado ao estudo.

\section{CONSIDERAÇÕES FINAIS}

Conclui-se que os discentes de uma forma geral apresentaram percepção positiva acerca do apoio recebido no estágio por parte dos professores orientadores. Além disso, demonstraram maior preocupação com a sua sobrevivência durante a realização dos estágios, o que é natural, considerando o momento de socialização profissional do estudante. Entretanto, a preocupação com a dimensão Impacto da Tarefa também foi presente para este grupo de alunos, o que pode estar associado ao desejo de que a atuação pedagógica durante o ECS não só tenha importância para o estudante universitário, mas também faça a diferença na vida dos alunos atendidos na Educação Básica.

Aotentar identificar os diferentes perfis de estudantes que realizavam oECS, observaramse três grupos distintos frente à motivação e preocupação dos acadêmicos. Destaca-se que aspectos associados à renda suficiente para atender as necessidades básicas dos alunos e tempo dedicado ao estudo foram preponderantes para compreender os comportamentos e sentimentos dos estudantes frente às atividades do ECS.

Notou-se que os estudantes do primeiro grupo eram desmotivados e tinham apenas preocupação em realizar as tarefas, o que pode estar associado ao processo de avaliação do aluno. Além disso, a falta de renda diminui as oportunidades dos discentes de migrar para outras cidades que ofertam os cursos de graduação de seu interesse. Portanto, o interesse inicial do universitário não é atendido. Assim, para possuir um título universitário, os discentes trabalham para ter condições de realizar o curso de graduação considerando suas condições financeiras e de tempo.

O segundo e terceiro grupos sofrem interferência do aspecto financeiro e do tempo de dedicação semanal de maneiras distintas. Os discentes do terceiro grupo tinham baixa preocupação, embora fossem motivados. 0 que pode estar associado ao tempo disponível para estudar, ofertado pela renda suficiente para sua manutenção nos estudos. Entretanto, os do segundo grupo eram preocupados e motivados frente às atividades do estágio, pois consideravam que este momento de formação é importante para aquisição de conhecimentos e habilidades que possibilitarão ter maior facilidade na sua atuação profissional. Destaca-se que a falta de tempo para o estudo pode ser um fator para a elevada preocupação, enquanto a motivação pode estar associada à renda, considerando que o ingresso na carreira docente pode favorecer os alunos na aquisição de melhores salários.

Como implicações práticas sugere-se que os docentes explorem com maior afinco nas suas disciplinas a aplicação de atividades, como: seminários, elaboração e aplicação de planos de aulas e elaboração de festivais com participação de alunos da educação básica. Tais ações colaborarão na minimização do efeito negativo do "choque com a realidade" ocorrido no aluno durante a realização da disciplina ECS. Além disso, expandir as atividades extracurriculares internas no curso de graduação, como a participação discente em grupos de monitoria 
esportiva, grupos de estudos e projetos de pesquisa voltados ao ensino podem ser estratégias para envolver os alunos em atividades relacionadas à docência.

\section{REFERÊNCIAS}

BARDAGI, Marucia Patta; HUTZ, Claudio Simon. Satisfação de vida, comprometimento com a carreira e exploração vocacional em estudantes universitários. Arquivos Brasileiros de Psicologia, v.62, n.1, p.159-170, 2010.

BRASIL. Instituto Brasileiro de Geografia e Estatística (IBGE). Cadastro Central de Empresas 2014. Rio de Janeiro, 2016. Disponível em: <http://cidades.ibge.gov.br/xtras/perfil. php?codmun=411350>. Acesso em: 05 jul. 2017.

BRASIL. Ministério da Educação. Instituto Nacional de Estudos e Pesquisas Educacionais Anísio Teixeira (INEP). ENADE 2014: Exame Nacional de Desempenho dos Estudantes. Relatório de área. Educação Física Licenciatura. Brasília, 2014. Disponível em: <http://download.inep. gov.br/educacao superior/enade/relatorio sintese/2014/2014 rel educacao fisica licenciatura.pdf $>$. Acesso em: 27 dez. 2016.

COSTA, Bruno Oliveira. Preocupações pedagógicas e desenvolvimento profissional em Educação Física: passo ou descompasso? 2013. 107 f. Dissertação (Mestrado) - Programa de Pós-graduação em Educação, Contextos Contemporâneos e Demandas Populares, UFRRJ, Seropédica, 2013.

FARIAS, Gelcemar et al. Preocupações pedagógicas de estudantes-estagiários na formação inicial em Educação Física. Motriz, n.3, v.14, p.310-319, 2008.

FIGUEIREDO, Zenólia Cardoso. Experiências sociocorporais e formação docente em Educação Física. Movimento, n.14, v.1. p.85-110, 2008.

FOLLE, Alexandra et al. Construção da carreira docente em Educação Física: escolhas, trajetórias e perspectivas. Movimento, v.15, n.1, p.25-49, 2009.

FULLER, Frances. Concerns of teachers: A Developmental conceptualization. American Educational Research Journal, v.6, n.2, p.207-226, 1969.

FULLER, Frances; BROWN, Oliver. Becoming teacher. In: RYAN, Kevin (Ed.). Teacher Education: The seventy-four Yearbook of the National Society for the study of Education. Chicago: University of Chicago, 1975. Pt.2, p.25-52.

GOMES, Patrícia; QUEIRÓS, Paula; BATISTA, Paula Maria. A socialização antecipatória para a profissão docente: Um estudo com estudantes de Educação Física. Sociologia, Revista da Faculdade de Letras da Universidade do Porto, v.28, p.167-192, 2014.

GRAÇA, Amândio Braga Santos. A construção da identidade profissional em tempos de incerteza. In: BATISTA, Paula; GRAÇA, Amâncio; QUEIRÓS, Paula. (Eds.). 0 estágio profissional na (re) construção da identidade profissional em educação física. Porto: Editora FADEUP, 2014. 
GUEDES, Dartagnan Pinto; SANTOS, Cintia Anira dos; LOPES, Cynthia Correa. Estágios de mudança de comportamento e prática habitual de atividade física em universitários. Revista Brasileira de Cineantropometria e Desempenho Humano, v.4, v.8, p.5-15, 2006.

GUIMARÃES, Sueli Édi Rufini; BUROCHOVITCH, Evely. O estilo motivacional do professor e a motivação intrínseca dos estudantes: uma perspectiva da teoria da autodeterminação. Psicologia: Reflexão e Crítica, v.17, n.2, p.143-150, 2004.

ISRAEL, Maria Natividade; DUARTE, Ana Maria. Motivação inicial para a profissão docente dos alunos do curso de Desporto e Educação Física da Universidade do Porto. Actas Congreso Galego-Portugués de Psicopedagogía, v.4, n.6, p. 919-926, 2000.

IZA, Dijnane Fernanda Vedovatto; SOUZA NETO, Samuel de. Os desafios do Estágio Curricular Supervisionado em Educação Física na parceria entre universidade e escolas. Movimento, v.21, n.1, p.111-124, 2015

JESUS, Saul Neves de. A motivação para a profissão docente: contributo para a clarificação de situações de mal-estar docente e para a fundamentação de estratégias de formação de professores. Aveiro: Estante, 1996.

JOLY, Maria Cristina Rodrigues Azevedo; PRATES, Eli Andrade Rocha. Avaliação da escala motivacional acadêmica em estudantes paulistas: Propriedades Psicométricas. Revista Psico USF, v.16, n.2, p.175-184, 2011.

LENS, Willy; MATOS, Lennia; VANSTEENKISTE, Maarten. Professores como fonte de motivação dos alunos: O quê e o porquê da aprendizagem do aluno. Revista Educação, v.31, n.1, p.17-20, 2008.

MARCON, Daniel; NASCIMENTO, Juarez Vicente; GRAÇA, Amândio Braga Santos. A Construção das competências pedagógicas através da prática como componente curricular na formação inicial em Educação Física. Revista Brasileira de Educação Física e Esporte, v.21, n.1, p.11-25, 2007.

MAROCO, João. Análise estatística com utilização do SPSS. 3. ed. rev. aum. Lisboa: Silabo, 2007.

MARTINS, Iguatemy Maria de Lucena. Intervenção profissional e Formação superior em Educação Física: Articulação Necessária para a Qualidade do Exercício Profissional. Rio de Janeiro: CONFEF, 2015.

MATOS, Zélia; GOMES, Paula Botelho; GRAÇA, Amândio Braga Santos; QUEIROZ, Paula A. A Valorização dos problemas em situação de estágio: Preocupações dos estudantes-estagiários e formadores. In: BENTO, J; MARQUES, A. (Org.). As ciências do desporto na escola. Porto: FCDEF, 1991.p.359-367.

McBRIDE, Ron E. Perceived teaching and program concerns among pre-service teachers, university superiors and cooperating teachers. Journal of Teaching in Physical Education, v.3, n.3, p.36-43, 1984.

NETO, Samuel de Souza; SARTI, Flávia Medeiros; BENITES, Larissa Cerignoni. Entre o ofício do aluno e o habitus do professor: os desafios do estágio supervisionado no processo de iniciação a docência. Movimento, v.22, n.1, p.311-324, 2016.

OLSEN, Dwayne G.; HEYSE, Kathy L. Development and concerns of first-year and reentry teachers with and without mentors. Annual Meeting of American Educational Research 
Association. Boston, 1990. Disponível em: <https://eric.ed.gov/?id=ED323188>. Acesso em: 02 maio 2017.

PARANÁ. Casa Civil. Decreto Estadual 1.647 de 15 de dezembro de 1999. Autoriza implantação dos cursos de Pedagogia e Administração na Faculdade Intermunicipal do Noroeste do Paraná. Diário Oficial do Estado do Paraná. Curitiba/PR, 1999.

QUEIRÓS, Paula. A construção da identidade profissional em tempos de incerteza. In: BATISTA, Paula; GRAÇA, Amâncio; QUEIRÓS, Paula. (Eds.). 0 estágio profissional na (re) construção da identidade profissional em educação física. Porto: Editora FADEUP, 2014.

RIBEIRO, Vandressa Teixeira et al. Preocupações pedagógicas e competência profissional de estudantes de educação física em situação de estágio. Revista de Educação Física/UEM, v.26, n.1, p.59-68, 2015.

SCAVONE, Lucila. Maternidade: transformações na família e nas relações de gênero. Revista Interface: Comunicação, Saúde e Educação, n.8, p. 47-59, 2001.

SILVA PIOVANI, Verónica Gabriela; BOTH, Jorge. Relaciones entre el tiempo de realización de práctica docente curricular y las preocupaciones pedagógicas de estudiantes de educación física de Uruguay. Educación Física y Deporte, v.33, n.2, p.225-248, 2014.

SILVA PIOVANI, Verónica. Gabriela; BOTH, Jorge; NASCIMENTO, Juarez Vieira do. Preocupaciones pedagógicas de los estudiantes de Educación Física de diferentes domicílios sociales de Uruguay. Movimento, v.18, n.4, p.77-98, 2012.

VIEIRA, Lenamar Fiorese; VIEIRA, José Luiz Lopez; FERNANDES, Renata. Competência profissional percebida: um estudo com estudantes de Educação Física em formação inicial. Revista de Educação Física/UEM, v.17, n.1, p.95-105, 2006. 